\title{
Die With Your Boots On : agressivité et exclusion dans La rage, de Louis Hamelin
}

\author{
Jean-Pierre Thomas \\ Université York
}

\begin{abstract}
Résumé
Où se situe la frontière qui sépare l'agressivité de la violence? Un faux pas risque-t-il d'entraîner d'un domaine dans l'autre? Le romancier québécois Louis Hamelin a exploré cette question dans son premier roman, La rage, paru en 1989. Puisant dans un registre intertextuel où affleurent quelques « slogans» heavy metal qui, tels de véritables leitmotive, ornent les soubassements de l'œuvre, l'auteur a placé son personnage principal dans un contexte où l'agressivité et l'exclusion deviennent les remèdes à la souffrance de vivre. Il s'agit ici d'examiner l'utilisation que l'écrivain fait des extraits de chansons cités, de déterminer jusqu'à quel point ceux-ci sous-tendent la trame narrative et d'évaluer l'effet qu'ils ont sur la constitution d'un imaginaire de l'agressivité.
\end{abstract}

\begin{abstract}
Where does the line that borders aggressiveness from violence rest? Can a faux pas drive from one realm to the other? French Canadian novelist Louis Hamelin has examined these questions in his first novel, La rage, published in 1989. Through a range of quotes where heavy metal "slogans" occupy a central place, the author has put his main character in a situation where aggressiveness and exclusion ease the pain of living. This paper examines how Hamelin uses the quoted songs; it tries to find if and to what extent they have an impact on the narrative; finally, it evaluates the effects they have on a potential imaginary of aggressiveness.
\end{abstract}


Rock. Le mot fait instantanément surgir des images qui, selon les époques ou les dispositions, laissent un arrière-goût irritant ou évoquent une atmosphère festive. Révolte, désordre, excès : voilà quelques-uns des substantifs qui sont souvent rattachés à la musique rock $^{1}$. La mauvaise presse qu'a longtemps récoltée ce phénomène musical n'est pas étrangère aux frasques qui en ont accompagné les origines. Se complaisant dans l'élaboration de modes d'expression considérés comme extrêmes, le rock s'est d'emblée donné pour mandat de servir la cause de la libération à l'égard des normes, ce qui l'a relégué dans les marges de communautés désireuses de poser des règles rigides. Comme toute action répressive entraîne tôt ou tard une réaction exaltée, le rock n'a pas manqué de répliquer, aidé de ses rejetons. L'un de ceux-ci, le heavy metal, passe pour être particulièrement indiscipliné ${ }^{2}$. Toute une imagerie binaire accompagne thématique, idéologie, voire philosophie metal, imagerie qui privilégie « les ambiances [...] volontiers sombres et morbides » (Hein, 2004, p. 46) et « la face sombre de la nature humaine » (Ibid., p. 162). Cette posture dresse entre les adeptes de ce genre musical et leurs pairs une frontière faisant d'eux des étrangers réciproques. Comme le remarque Keith Kahn-Harris :

\footnotetext{
${ }^{1}$ Voir, à ce propos, l'analyse de Deena Weinstein, 2000, p. 237-275.

${ }^{2}$ Bien que ce que d'aucuns nomment aujourd'hui heavy metal ne corresponde plus tout à fait au genre musical qui est apparu dans les années 1970 et qui, à partir de la deuxième moitié de la décennie suivante, s'est segmenté en une multitude de sous-genres, j'utiliserai ici cette expression générique par commodité.
} 
« Metal divides people. $»^{3}(2007$, p. 1) L’altérité qui surgit conjointement marque ceux qui se réclament du heavy metal du sceau d'une différence qui, pour ne pas nécessairement les transformer en parias, n'en demeure pas moins souvent vecteur d'exclusion.

L'on est en droit de se demander si le constant rapprochement du metal avec la violence, auquel se sont voués partisans des critiques et progressiste et conservatrice, est justifié. D’ailleurs, où se situe la frontière qui sépare la pulsion agressive - contenue dans les limites d'une colère cathartique ou d'une aigreur sublimée-de la violence irrépressible? Le défoulement institué par le recours aux topoï du phénomène rock devientil forcément à un moment incitation à la transgression? Peut-être la rage qui surgit en un individu confronté aux répressions imposées par les institutions trouve-t-elle dans le heavy metal un exutoire sain; à moins que ce genre musical, authentique bastion de la révolte, n'accentue plutôt en ceux qui s'adonnent à son écoute la volonté de refus. Voilà quelquesuns des questionnements que soulève la lecture de La rage, roman de l'auteur québécois Louis Hamelin, paru en 1989 et récipiendaire la même année du prix du Gouverneur général du Conseil des Arts du Canada. Puisant dans un registre intertextuel ${ }^{4}$ inspiré par

\footnotetext{
${ }^{3}$ Je traduis : « Le metal divise les individus. »

${ }^{4}$ La plupart des commentateurs qui se sont attaqués à l'analyse du roman de Hamelin se sont fait forts de relever le haut apport intertextuel/interdiscursif qu'on y trouve. André Lamontagne, dans un texte portant principalement sur ce phénomène, parle d'un narrateur qui s'abandonne à une " frénésie citationnelle en convoquant une multitude d'intertextes et d'auteurs [...], une intertextualité proliférante et dispersée [qui] informe le discours du narrateur». (2004, p. 183) Le critique va jusqu'à poser l'hypothèse que "l'intertextualité s'affirme comme figure modélisante du roman, comme fonction régulatrice d'un discours idéologiquement dispersé » (Ibid., p. 184), bref, comme soubassement de l'œuvre. Cela dit, bien que Lamontagne note la présence d'un intertexte musical rock, il le fait à la volée et n'y accorde qu'une importance mineure. En outre, il rattache exclusivement le phénomène à " des réalités américaines » (Ibid., p. 196), alors que, nous le verrons, l'américanité n'est ici que fort passagère - à ce propos, François Paré évoque de son côté des « réseaux extrêmement complexes d'intertextes divers et de références culturelles nombreuses, québécoises, américaines et françaises, entre autres ». (Ouellet et Paré, 2008, p. 22) Michel Nareau et Jacques Pelletier indiquent pour leur part que «cette œuvre se distingue, c'est l'une de ses constantes, par une intertextualité foisonnante, ramifiée, parfois latente, parfois affichée de manière plus ostentatoire » $(2015$, p. 9), tandis que Valérie Ménard a démontré à quel point les réseaux intertextuels s'imposent chez les auteurs de la génération X (à laquelle Hamelin appartient). Nonobstant ces travaux, aucun spécialiste ne s'est vraiment intéressé à la question du rôle et de l'impact du heavy metal dans La rage.
} 
certains «classiques» du rock, le romancier a placé son personnage principal dans un contexte où le défoulement devient le remède à la souffrance de vivre. Quelques « slogans » metal particulièrement prisés ornent l'œuvre et s'orchestre progressivement une intrigue qui oscille entre agressivité et violence. Il s'agira au cours des prochaines pages d'examiner l'utilisation que fait l'écrivain de ces formules, de déterminer jusqu'à quel point elles sous-tendent la trame narrative et d'analyser l'effet qu'elles produisent sur la lecture du roman. La réflexion abordera par la suite la question de l'imaginaire de l'exclusion et de ses relations avec la violence pour établir si, au sein d'un univers marqué par l'ostracisme, la musique heavy metal, en tant que catalyseur de transgression, constitue un apport positif dans la quête de liberté de l'individu.

\section{Un roman à " consonance " metal}

Le personnage principal du roman, Édouard Malarmé, après avoir terminé des études de baccalauréat en biologie, rompt pratiquement toute attache sociale et devient squatter. Installé dans un chalet abandonné de Saint-Canut, dans les Laurentides, il fomente de fantasques projets iconoclastes ayant pour cible l'aéroport de Mirabel, sis tout près. En compagnie de son ami rocker et motard Johnny, il fréquente régulièrement un bar de la région, le Pullford Lodge, et s'y livre à ses activités coutumières : jouer au billard et au pinball, boire de la bière et écouter de la musique heavy metal. La rage guette Malarmé, un renard l'ayant mordu. Cette rage est aussi celle qu'il ressent au contact d'une société oppressive qui l'empêche de s'exprimer. Et Malarmé rumine, il enrage, jusqu'au moment où il laisse libre cours à ses sentiments ravageurs : le meurtre du propriétaire du chalet qu'il habite le fait basculer dans la violence.

D’où provient la colère éprouvée par Malarmé? Très tôt dans sa vie, le personnage principal de La rage a senti sourdre en lui un désir de non-conformité. Rebelle, il s'en 
prend çà et là dans le roman aux institutions qui, selon lui, empêchent l'individu d'aspirer à l'autonomie. La description physique du jeune homme permet de repérer un type précis, celui du voyou peu respectueux des tendances esthétiques de son époque : «Je suis vêtu d'un pantalon ample et kaki, avec des pièces de cuir aux genoux, d'un T-shirt noir me découvrant les bras jusqu'aux épaules, et un bandeau rouge m'enserre le front et discipline une chevelure plutôt longue, bouclée et effrontée ». (Hamelin, 1989, p. 945) Force est de voir en Édouard Malarmé un jeune adulte qui a rompu avec les normes. Comme il le prétend lui-même, il a «sauté la clôture. À bas les poteaux de la culture!» (128) Ayant « pris le parti de tout refuser » (366), s'étant tout jeune abstenu de répéter mécaniquement les paroles préprogrammées du culte religieux (194), se voyant sous les traits d'un « ennemi public numéro zéro, cheval de Troie dans [un] dragon de béton » (270), Malarmé est un «facteur de ralentissement» (215) - d'abord dans sa propre famille, ensuite au sein du corps social. Qu'il s'agisse de fracasser sans raison la vitrine d'un commerce lors d'une escapade à Laval ou de faire l'amour avec une compagne de passage dans un recoin de l'aéroport de Mirabel, Malarmé savoure l'illicite. D'où lui vient cette prédilection? Il s'explique : «je suis venu au monde déjà irréductiblement retranché dans un refus bien net de la vie bien blanche du dehors ». (182) Sa naissance apparemment forcée, marquée par la résistance à l'égard d'un monde extérieur aseptisé, semble le prédestiner à vivre à l'écart. L'on ne se surprendra guère de le voir fréquenter un milieu rythmé par une musique ellemême longtemps considérée comme hors-normes.

Le petit noyau d'amis qui se forme autour de Johnny, comparse de Malarmé, se complaît dans les esclandres aux implications douteuses - chasse au canard plus ou moins

\footnotetext{
${ }^{5}$ Les références au roman de Louis Hamelin seront dorénavant insérées sans nom d'auteur ni année de publication, directement à la suite de l'extrait cité.
} 
www.revue-analyses.org, vol. 14, n², automne-hiver 2019, p. 86-111.

légale ou virées alcoolisées. Cela dit, même au sein de ce groupe qui constitue sa seule véritable famille, Malarmé fait office d'inadapté, car il a la tête dans les nuages, il aime philosopher, ce qui le sépare de ses amis. Marginal il est, lui qui, pour adopter la définition de Martine Xiberras, «se [met] hors du système du fait d'une inadaptation quelconque : il est aux confins, à la périphérie ou en tout cas au-delà de la ligne d'horizon ». (1993, p. 122) Étranger au monde qui l'entoure, Malarmé apparaît comme un exclu au sein d'un groupe d'exclus. L'exclusion, dont Xiberras indique qu'elle implique «le rejet hors des représentations normalisantes de la société » (p. 25), convie à « une rupture du lien [...] qui attache fidèlement ou normativement les acteurs sociaux au modèle de la société » (p. 26), question que j'explorerai sous peu en la mettant en rapport avec la musique écoutée par ce petit gang. Toujours est-il que la rage guette Malarmé, qui choisit de « renaude[r] au lieu de prier. » (251)

Quel rôle le heavy metal joue-t-il dans ce roman aux couleurs sombres? Circonscrivons d'abord l'utilisation que fait Hamelin du phénomène. Les références au metal sont nombreuses dans La rage, mais la musique n'est pas le seul motif à étayer une intrigue fondée sur le désir de Malarmé de renverser toute forme d'autorité. Le metal n'en joue pas moins ici un rôle fulgurant ${ }^{6}$. Dès l'abord, le narrateur se dit habité par « une musique de fond conquérante, sous [s]on crâne » (31), dont les effets ne se démentiront à aucun moment. Mais il y a plus : le metal colore littéralement l'univers dans lequel le jeune homme vit. Lors de la visite de son ami Johnny, qui fait résonner dans le petit chalet la musique de groupes « Heavy Métal gothique » (208), le lieu se transforme : «Aux accents de ces incantations chthoniennes, le petit castel du pied de la pente durcie essaie de devenir

\footnotetext{
${ }^{6}$ Bien qu'allusion soit faite au metal dans pratiquement toutes les parties du roman, c'est surtout au cœur du deuxième chapitre que la musique occupe une place prépondérante.
} 
un sombre et sinistre château où les ombres veulent nous avaler [...]»(208), explique le narrateur, qui s'avoue fasciné par l'imagerie repérée dans cet univers mystique :

Je me suis [...] perdu dans la contemplation du boîtier de la cassette qui jouait à ce moment, produit d'un groupe que je ne connaissais pas, les RAGING DOGS. Sur le dessin $[. .$.$] , on voyait une espèce de mutant poilu revêtu d'une armure de chevalerie$ éclatante, et de dessous le heaume au ventail relevé, une gueule à la dentition terrifiante dardait ses crocs baveux tandis que des yeux rouges luisaient dans l'ombre. On devinait des meutes de loups, silhouettées à travers les bois squelettiques. En arrière-plan, un château médiéval stagnait dans un halo de brumes noirâtres traversées d'éclairs, élevant une tour sombre à la rencontre du ciel menaçant ${ }^{7}$. Je dis à Johnny :

- Tu me fais écouter de drôles de choses, mon vieux.

- Ça te fait du bien, Eddy, fut sa réponse distraite. (211)

Initié par son compagnon aux accents fantastiques du heavy metal, Malarmé y découvre une force insoupçonnée. Le Pullford Lodge, où les amis assistent aux prestations de groupes metal locaux, se métamorphose du bistro malpropre qu'il est habituellement en « une cathédrale à la nef balayée par l'athéisme » (45), où « le tonnerre métallique d['une] musique [...] Heavy Rock d'une brutalité inouïe (et pourtant très éloignée d'être inaudible) et d'inspiration ostensiblement satanique»(45-46) se déverse sur les consommateurs exultants. Quant aux musiciens, ils figurent «[t]rois disciples de Lucifer [qui] continuaient à invoquer à grands râles, là-devant, les mauvais esprits, les racailles et les engeances moyenâgeuses leur servant de modèles et de muses » (50) dans la préparation de « leur véhémente théurgie de bas-fonds. » (50) Messe postmoderne que celle-là, qui rend compte d'une resacralisation du réel par voie de subversion ${ }^{8}$.

\footnotetext{
${ }^{7}$ Il serait probablement juste de relever ici la valeur de préfiguration de ce dessin qui, par le personnage du mutant ainsi que la tour mis en scène, annonce l'assaut de Malarmé sur la tour de contrôle de l'aéroport, à la fin du roman. La fascination du protagoniste pour les tonalités sombres véhiculées par l'univers décrit n'a donc rien pour surprendre.

${ }^{8}$ À propos d'un rapprochement possible entre musique metal et sacré, voir Alexis Mombelet, «La musique metal : des éclats de religion et une liturgie. Pour une compréhension sociologique des concerts de metal comme rites contemporains ", Sociétés. Revue des sciences humaines et sociales, n 88, 2005/2, p. 25-51.
} 
www.revue-analyses.org, vol. 14, n², automne-hiver 2019, p. 86-111.

Parlant de Lucifer, les topoï du genre metal sont omniprésents dans le roman : la musique, bien entendu, mais aussi le cuir, les bracelets à clous, la femme fatale à poitrine protubérante, l'alcool et la drogue (que consomment le groupe d'amis), les motocyclettes vrombissantes, l'attitude machiste, les cheveux longs, et j'en passe. Stéréotypes marqués par une époque précise (les années 1980, moment où se déroule le roman), ces lieux communs surdéterminent le thème de la rage. Et si le metal redonne vie aux lieux (jusqu'aux vieillards qui se mettent à frétiller au son de la musique!), c'est que le message transmis dans les extraits de pièces citées empreint l'atmosphère d'une teneur électrisante.

\section{Un metal de circonstance}

Avoir choisi la musique et l'imagerie heavy metal comme toile de fond marque La rage d'une couleur singulière. Si les topoï font partie intégrante de la trame, la musique comme telle ne laisse pas sa place. Évidemment, le roman ne réussit pas à rendre la sonorité metal, sinon de manière détournée, en utilisant ces substituts que sont les mots. Le code metal défini par Deena Weinstein-composé d'une sonorité qui exulte la puissance, d'une dimension visuelle rendue par les topoï mentionnés ci-haut ainsi que d'un registre verbal caractérisé par le recours à une thématique binaire (où l'expérience dionysiaque s'oppose au chaos) (Weinstein, 2000) - apparaît donc ici de façon elliptique. L'aspect verbal m'intéressera davantage, compte tenu que de nombreux extraits de morceaux, insérés à même plusieurs passages du roman, scandent certains hymnes metal. L'action se déroulant en 1983, c'est le heavy metal du début de la décennie qui se trouve exploité. Ce moment est marqué par l'avènement de ce que les critiques musicaux ont nommé la New Wave of British Heavy Metal. Sous l'égide de groupes tels que Judas Priest et Iron Maiden, le genre se développe, peu après sa naissance dans les années 1970 - avec ces pionniers qu'ont été

Led Zeppelin et Black Sabbath. L'engouement pour le metal se transmet bientôt à 
JEAN-PIERRE THOMAS, « Die With Your Boots On : agressivité et exclusion dans La rage, de Louis Hamelin »

l'ensemble de l'Europe, puis l'Amérique du Nord emboîte le pas. Il n'est dès lors guère surprenant que Hamelin ait misé sur les paroles de chansons de groupes metal anglais (Iron Maiden), américain (Quiet Riot) et allemand (Accept), qui tous exemplifient l'essence du metal tel qu'il apparaît à l'époque.

L'auteur incorpore apparemment de façon aléatoire des extraits de pièces dans son texte, mais le procédé se systématise rapidement. La lettre majuscule lui sert à insérer a) des onomatopées transposant des bruits tonitruants (boules de billard qui s'entrechoquent sur la table de jeu ou ahans de Malarmé en train de fendre du bois) et b) des paroles de chansons. La stratégie est utilisée à outrance et les passages en lettres majuscules se superposent le plus souvent aux pensées du narrateur, dont ils interrompent le fil. Il s'avère ainsi difficile de distinguer la simple onomatopée du segment de chanson, témoin l'extrait suivant où le narrateur, décrivant la scène où il fait la connaissance de Johnny, s'apprête à jouer une partie de billard au son des Brains Blasters interprétant une pièce du groupe Accept :

Le chanteur s'époumonait sur scène, la glotte pratiquement arrachée de ses gonds. WATCH THEM THEY'RE GONNA BREAK THEIR CHAINS HEY! CHALK A TALC A POM POM PLAQUE. Le début fracassant du sieur Johnny, qui envoya promener les boules bariolées aux quatre coins de la table, se voulait un sérieux coup de semonce. (54)

Connaître les paroles de la pièce «Balls to the Wall» est nécessaire pour pouvoir retrancher les onomatopées du segment. Je me restreindrai dans le cadre de cette analyse aux passages cités d'une pièce d'Accept, de deux pièces d'Iron Maiden et d'une autre de Quiet Riot ${ }^{9}$. Les extraits cités n’ont assurément pas été choisis au hasard, car ils aiguillent le rapport qui se crée avec le metal sur le plan discursif. Aucun titre de chanson n'étant

\footnotetext{
${ }^{9}$ Bien que d'autres fragments de chansons soient disséminés çà et là dans le texte (par exemple, «Back in Black» et « Hells Bells » du groupe australien AC/DC), les extraits choisis sont ceux qui reviennent le plus souvent et qui semblent avoir un effet marquant sur la diégèse.
} 
www.revue-analyses.org, vol. 14, n², automne-hiver 2019, p. 86-111.

indiqué dans le texte, le lecteur non averti risquera de ne pas décoder la provenance des extraits. Conséquence : il ne percevra pas la finesse avec laquelle Hamelin choisit ses fragments et les recompose.

Le premier passage dont le romancier se sert provient de l'album Balls to the Wall, que le groupe Accept a fait paraître en 1983 et dont la pièce éponyme, sorte d'hymne prêchant les droits de la personne et la rébellion, est devenue la plus connue du groupe. Outre le passage cité précédemment, Hamelin insère le vers clé du refrain, précédé d'une courte série d'onomatopées: «KEY PLOW BAM BALLS TO THE WALL MAN YOU'LL GET YOUR BALLS TO THE WALL » (55). Puis l'écrivain reprend les deux extraits cités, qu'il recompose toutefois en partie, histoire de les enrichir ou d'en inverser le sens : « BAMB BACK POÏNG WATCH THEM OH THEY'RE GONNA BREAK THEIR BACK BLACK POÏNG » (55) - les chaînes à briser sont remplacées par le dos, ce qui accentue les difficultés qu'implique la rébellion -, « BAN BOMB BALLS TO THE WALL MAN YOU'LL GET YOUR BALLS TO THE BAAL »(56), et enfin «BAC BONN WHAM WALL TO THE BALLS MAN WALL TO THE BAÏL » (56). La pièce, entendue au premier degré, procède à une apologie de la destruction, orchestrée par les martyrs dont les institutions ont trop longtemps abusé. L'auditeur de la chanson sera invité à se joindre à ces opprimés pour aider à libérer le monde des excès de l'asservissement. Le carnage célébré est soutenu chez Hamelin par l'espoir en une recréation éventuelle, ce qu'illustrerait l'allusion au dieu ougaritique Baal, reconnu pour son combat afin d'ériger un monde fécond. Destruction et création vont donc de pair. L'inversion de «Balls to the Wall» en « Wall to the Balls » indique probablement pour sa part que le renversement qui se prépare aura pour conséquences d'instaurer un nouvel ordre reposant sur une transvaluation des valeurs, la matière répondant aux coups orchestrés par le corps. La seconde thématique 
metal joue ici à plein. "The discourse on chaos in heavy metal lyrics includes interest in disorder, conflict, opposition, and contradiction. [...] It speaks of injustice and of resistance, rebellion, and death $»^{10}$, selon Deena Weinstein. (2000, p. 39) Cette posture oppose les forces en présence, mais place le point de vue dominant du côté du dominé, histoire de proposer une inversion possible. "[Metal] symbolizes a refusal to conform to rules of respectability that repress vitality or to submit to those who wish to exert their domination over others. $»^{11}$ (Ibid., p. 42) La chanson s'inscrit à merveille dans la logique d'un récit où le personnage principal rêve de renverser les institutions en place.

Le deuxième extrait cité provient du morceau du groupe Iron Maiden intitulé « Flight of Icarus », tiré de l'album Piece of Mind (paru en 1983). Il s'agit d'une adaptation libre du récit mythologique grec où Icare et son père Dédale réussissent à se tirer du labyrinthe crétois grâce à des ailes collées à leurs bras à l'aide de cire. Icare se rapproche du soleil, la cire fond et il s'écrase au sol. Le récit a été transformé par le groupe britannique, Icare n'étant plus secouru par son père mais bien trompé par celui-ci, qui le pousse vers une mort certaine. Le rôle joué par Dédale dans la pièce confère au mythe une tournure particulière : l'accent est mis sur le conflit des générations, qui se solde par la trahison du père à l'égard de sa progéniture ${ }^{12}$. L'imagerie binaire du texte rend évidente la problématique de l'exclusion. Comme les enfants risquent par leurs rêves de faire éclater le monde construit par les adultes, ces derniers préfèrent les éliminer en les encourageant au

\footnotetext{
${ }^{10}$ Je traduis : «La thématique du chaos dans les paroles de chansons heavy metal recoupe un intérêt pour le désordre, le conflit, l'opposition et la contradiction. Elle traite d'injustice et de résistance, de rébellion et de mort. »

${ }^{11}$ Je traduis : «Le metal symbolise un refus de se conformer aux règles qui répriment la vitalité ou de se soumettre à ceux qui souhaitent exercer une domination sur leurs pairs. »

${ }^{12}$ Le chanteur d'Iron Maiden, Bruce Dickinson, a apparemment avoué avoir modifié le récit afin d'en faire une allégorie de la rébellion des adolescents contre l'autorité adulte (celle-ci ayant causé la mort d'Icare dans la chanson). Source : http://en.wikipedia.org/wiki/Flight_of_Icarus, 7 mai 2019.
} 
www.revue-analyses.org, vol. 14, n², automne-hiver 2019, p. 86-111.

vol : «FLY ON YOUR WAY LIKE AN EAGLE FLY AS HIGH AS THE SUN » (57). Le soleil, figure patriarcale dans la mythologie de plusieurs traditions anciennes, interrompt l'avancée d'Icare, comme le père d'Édouard Malarmé l'a empêché d'accomplir ses rêves d'enfant. L'extrait se transforme sous la plume de Hamelin : «BLACKABACK AS HIGH AS THE SUN CARE CARE ICARE BALLS TO THE SUN MAN » (57). Malarmé rêve durant tout le roman de prendre son envol, lui qui s'immisce par la pensée à bord des Jumbo Jets le survolant et qui à la fin s'affuble du titre de « Renard-des-Airs » (404). Il était prédestiné au vol, mais aussi, en raison de la trahison paternelle tôt consommée, à la chute, à voir ses Balls quitter le mur pour brûler au soleil. L'élan qui l'habite génère une rage destructrice qui se concrétisera dans la violence, ce que la pièce d'Iron Maiden annonce : s'opposant à la foule, Icare prend son envol et monte, malgré les risques, à l'assaut de ses aspirations. Emblématique du sort qui attend le rebelle, la mort d'Icare figure le refus de la jeune génération de prendre en charge les projets de la précédente. Ce morceau a visiblement été choisi pour le message de dissidence qu'il transmet : le transfert symbolique de la responsabilité de la chute du fils au père reflète à merveille la rage qui habite Malarmé.

Avec l'extrait de Quiet Riot, on demeure dans le registre de la révolte, mais quelques nuances s'imposent. «BANG YOUR HEAD BUM BOUDDHA BUM BUM » (59), écrit Louis Hamelin. La pièce « Metal Health », tirée de l'album du même nom paru aussi en 1983, est vite devenue un autre hymne apprécié des amateurs de metal. Vu par certains comme le morceau qui a permis au heavy metal d'entrer dans la culture de masse mais qui n'en parle pas moins nommément de la sous-culture des headbangers -, « Metal Health », en jouant sur l'oxymore santé / folie, se livre à l'éloge de la différence et incite les jeunes non-conformistes à faire partie de la bande, au grand dam des adultes qui ne voient 
pas que se frapper la tête jusqu'à la folie est un signe de santé. Encore une fois, Hamelin semble n'avoir pas procédé à un choix aléatoire : par son contenu, la chanson appuie la démarche de son personnage, et par l'entremise d'un « BANG YOUR HEAD BOMBOMB BANG YOUR BLACK A BACK BALLS »(61), c'est le corps entier du rebelle qui participe à la révolte. Si la tête, centre de toute activité neurophysiologique, tout autant que les balls (référence aux parties génitales), sources de la fécondité masculine, doivent être frappées pour que le jeune révolté fasse valoir son existence aux yeux des oppresseurs ${ }^{13}$, c'est dire que le mouvement de subversion doit, par son recours à des principes hédonistes fracassants, affirmer la valeur hautement organique de l'existence. Il semble y avoir un transfert provisoire de la thématique du chaos à celle de la festivité. La transgression est toujours au rendez-vous (le chanteur Kevin DuBrow incitant dans la pièce le headbanger à se séparer de ceux qui ne font pas partie du groupe des élus), mais il s'agit d'une transgression qui vise une resacralisation par voie de carnavalisation, par une inversion des valeurs instituées. Cela dit, Dionysos ne se débarrassera pas de Chaos sans coup férir.

« Die With Your Boots On » est une autre pièce d'Iron Maiden citée par Hamelin, à quelques reprises d'ailleurs puisqu'il en fait la chanson fétiche de Johnny. Le refrain, seule partie mentionnée, se présente comme suit: «IF YOU'RE GONNA DIE IF YOU'RE GONNA DIE IF YOU'RE GONNA DIE DIE WITH YOUR BOOTS ON». (96) L'atmosphère du morceau est sombre. Il y est fait état de la figure du prophète de malheur, qui prédit moult catastrophes auxquelles l'humanité ne pourra échapper. Au dire de Paul Stenning : « The title was formed from a phrase whose origin is unknown but was probably

\footnotetext{
${ }^{13}$ Par « oppresseurs », entendons, dans le cadre du roman, autant l'État, représenté par les fonctionnaires du gouvernement intéressés à exproprier les habitants de la région décrite, que le propriétaire véreux qui ne laissera pas à Malarmé l'occasion d'occuper les lieux sacrés choisis par lui. Mais peut-être l'oppresseur par excellence est-il le père du protagoniste, en qui toute figure d'autorité se reflète presque directement.
} 
www.revue-analyses.org, vol. 14, n², automne-hiver 2019, p. 86-111.

linked to the military. The connotation would appear to be if you're going to go down, go down fighting. Better to die on the field of battle, in a blaze of glory, than in your bed. ${ }^{14}$ (2006, p. 90) Le thème principal du morceau est évidemment la mort, une mort qui, aussi inéluctable puisse-t-elle donner l'impression d'être (le propos est imprégné d'un mysticisme qui rappelle les paroles de certains prophètes bibliques), finit par pouvoir s'apprivoiser - ainsi se termine le dernier couplet.

Quels effets la présence de ces extraits exerce-t-elle sur la trame du roman? Parlons d'abord d'un impact catalyseur d'agressivité. Johnny plus que tout autre est victime de « l'animosité que la musique nourri[t] d'énergie brute » (210), lui qui trame l'assassinat du « bonhomme Bourgeois », propriétaire du chalet habité par Malarmé, au son d'Iron Maiden proclamant que la vie se goûte au présent. La frontière qui sépare l'agressivité de la violence se rétrécit à mesure que l'action progresse et, vers la fin du roman, Johnny, incité peut-être par cette musique qui lui est chère, aussi par la drogue mêlée à son sang, puis par ses rapports troubles avec les autorités, fonce délibérément à motocyclette dans un pilier de béton. Sa mort devient un acte de célébration de la violence libératrice - Johnny meurt « with [his] boots on ».

Mais ce défoulement d'émotions n'est pas le seul effet de la musique sur le comportement des personnages. La rage qui empreint le roman provient en partie de la vision du monde transmise par le metal. Quand onomatopées et paroles de chansons s'entremêlent, c'est presque toujours dans l'optique d'un coup assené à la vie : coup de queue de billard sur les balls colorées qui volent dans toutes les directions, mais aussi coup

\footnotetext{
${ }^{14}$ Je traduis: «Le titre tire son origine d'une phrase dont la provenance est inconnue, mais qui est probablement liée au domaine militaire. La signification semble en être que s'il faut connaître la défaite, autant se battre jusqu'au bout. Il vaut mieux mourir sur le champ de bataille, dans une envolée glorieuse, que dans son lit. »
} 
de gueule contre les institutions absolutistes. Malarmé, «fouetté [...] par la prestation des Kids du Heavy à la direction musicale » (63), sent monter en lui une rage sourde qui lui permet d'accomplir des exploits sur la table de billard. Cette énergie caractérise précisément la musique metal. Dès que celle-ci cesse, Malarmé rate ses coups et perd la partie. L'association manifeste entre musique et puissance d'action ne laisse aucun doute sur l'efficace du metal qui cravache l'auditeur. C'est comme si l'utilisation d'extraits de chansons metal préparait dans le livre la montée de la rage, une même ambivalence (sursaut d'agressivité et défoulement) étayant les deux phénomènes.

Semblable au metal lui-même, le discours sur le metal donne accès à des régions spécifiques du réel, différentes de celles qu'exploite habituellement tout un chacun. Comme l'explique Harris M. Berger :

In daily life, rage, fear, or sadness erupt because of negative events, real or imagined. In the framed sphere of music or literature, however, we are able to experience these emotions without having to suffer the consequences. Experiencing rage without actually having to suffer humiliation or violence [...], we are freed up to attend to the affect itself and treat the affect as an aesthetic object on its own terms..$^{15}$ (Berger, 1999, p. 272)

Cette musique teintée de violence convient à l'atmosphère de frénésie qui marque l'œuvre. Après que les « heavy mentals » (62) aient chanté la mort à tout vent, celle-ci jouxte la vie d’Édouard Malarmé, suscitant dans son esprit une vision de la totalité. Comment expliquer autrement l'intérêt porté au côté sombre de l'existence? Le metal peut écraser l'auditeur (une escapade au Pullford Lodge laisse les « tympans trépanés » [51], tandis que le « stéréo satanique [de Johnny] essaie de [...] jeter des sorts » [125]), mais il nourrit également

\footnotetext{
${ }^{15}$ Je traduis : « Dans la vie quotidienne, rage, peur ou tristesse surviennent en raison d'événements néfastes, réels ou imaginés. Dans la sphère close de la musique ou de la littérature, nous avons la possibilité de faire l'expérience de ces émotions sans avoir à en subir les conséquences. Faire l'expérience de la rage sans avoir à vivre l'humiliation ou la violence nous rend libres de prendre en compte l'affect et de le considérer comme objet esthétique en soi. »
} 
www.revue-analyses.org, vol. 14, n², automne-hiver 2019, p. 86-111.

l'imaginaire d'individus qui nécessitent la purgation d'un trop-plein d'émotions refoulées,

si bien que les «damnés [venus se placer] sous la protection tutélaire de leurs idoles de fer » (46) jubilent lorsqu'ils subissent « les exhortations méphistophéliques d'un groupe de suppôts du Heavy ». (47) Opposition intéressante, qui entraîne toutefois le narrateur dans une situation d'exclusion.

\section{De la dérive à l'exclusion}

L'utilisation que fait Hamelin du phénomène heavy metal a fort à voir avec la dimension transgressive du genre musical. L'action du roman se déroule en 1983, moment où le metal se dirige en Occident vers une polémique qui verra le PMRC (Parent's Music Resource Center) ${ }^{16}$ prendre des mesures draconiennes afin d'en limiter l'écoute chez les adolescents américains, mais La rage ne paraît qu'en 1989. Les six années qui séparent l'action du roman de sa parution ont vu le metal s'inscrire peu à peu dans la culture de masse et, simultanément, se constituer en une sous-culture autonome et légitime. La dimension transgressive qui le caractérisait à ses débuts continue toutefois d'être présente et le metal en récoltera un visage hors-la-loi, comme le suggère Jeffrey Jensen Arnett :

Violence, defying authority, breaking the norms and rules of society - these are common themes in heavy metal songs, and it could be argued (as the critics do) that these themes promote behavior that is disruptive to the rest of society, behavior such as violence toward self and others, sexual promiscuity, drug use and drunkenness, and the destruction of property. ${ }^{17}(1996$, p. 77$)$

\footnotetext{
${ }^{16}$ Le PMRC, fondé en partie par les épouses de sénateurs américains, s'est donné pour mandat de soustraire les adolescents à l'influence de certains genres musicaux (principalement le heavy metal et le rap). Pour la petite histoire du PMRC, voir Walser, 1993, p. 137-171. Au Québec, le Père Jean-Paul Regimbal a entretenu durant quelque temps la polémique, notamment avec la parution en 1983 de son livre Le Rock ' $n$ ' Roll - Viol de la conscience par les messages subliminaux.

${ }^{17} \mathrm{Je}$ traduis : " Violence, affront à l'autorité, transgression des normes et règles de la société - tels sont les thèmes récurrents dans les pièces heavy metal, et il pourrait être tentant de poser l'hypothèse (à l'instar des critiques) que ces thèmes encouragent des comportements dommageables pour l'ensemble de la communauté, comportements confinant à la violence à l'égard de soi-même et d'autrui, à la promiscuité sexuelle, à la consommation de drogue et d'alcool, au non-respect de la propriété d'autrui. »
} 
Transgression : «franchir une ligne », pour suivre la pensée de Gilbert Durand (1979, p. 24). Cette traversée de l'interdiction dynamise le rapport qui s'établit entre producteurs et consommateurs de musique heavy metal, les uns répondant à l'appel lancé par les autres. Comme quantité de formes d'arts, le metal vise à satisfaire la propension toute naturelle de l'individu à la liberté. Dans une société libre, cette musique n'a plus de sens. Il faut donc une possibilité de liberté ainsi que des instances brimant cette possibilité pour que le heavy metal ait sa place dans une culture. Le metal trouve sa raison d'être dans des sociétés où la liberté n'est pas un fait acquis. Comme il reste de la place pour l'interrogation et les changements, le metal se donne pour mandat de participer au processus de transformation de la communauté, accentuant la tension qui entretient le rêve utopique d'une société égale. Toléré par les autorités en raison de son pouvoir cathartique, il permet aux jeunes de célébrer leur passion de la vie par voie de défoulement, ce qui aide à conserver l'équilibre dans certaines strates sociales. Survient toutefois toujours le cas où le message perçu provoque une dérive, une errance, voire une déviance par rapport aux normes établies par le groupe social. La rage présente une telle situation: violence, défi jeté aux figures d'autorité, transgression des normes de la société, voilà autant de traits qui, dans l'œuvre, manifestent un désir de franchissement des frontières.

Le roman de Hamelin est imprégné de transgressions. Détail important: ces dernières entretiennent le plus souvent avec la musique un lien prégnant. Les vieillards qui se rendent au Pullford Lodge aspirent à prouver aux jeunes du coin « que ce n'est pas parce qu'on a passé quatre-vingts ans qu'on est condamné à l'ignorance de la bonne musique et des bonnes choses de la vie et que surtout, ce n'est pas parce qu'on est vieux qu'on devrait être empêché de pécher. » (50-51) Cette « bonne musique » aurait-elle obligatoirement à 
www.revue-analyses.org, vol. 14, n², automne-hiver 2019, p. 86-111.

voir avec le péché? Le Pullford Lodge accueille ceux qui souhaitent «vendre leur âme au diable » sous « une douche de brûlants décibels sortis tout droit du soufflet de Vulcain. » (47) L'on peut parler d'un éclatement des normes sociales (par voie d'excès sonores, moraux, esthétiques...) lesquelles trouvent leur contrepartie dans la musique, mais aussi dans les activités auxquelles celle-ci incite (billard, bagarre, drogue...) ainsi que dans les sentiments dont elle provoque l'apparition (agressivité, pulsion destructrice, volonté de puissance). Plus souvent qu'autrement, dans La rage, la mort accompagne cet éclatement.

Édouard Malarmé est habité par un désir de révolte qui l'entraîne à laisser se manifester son agressivité, verbale d'abord, physique ensuite, ce qui culminera en une irruption de violence incontrôlable, aux implications non plus simplement individuelles, comme dans le cas de l'agressivité, mais collectives, signe que la transgression aura été remplacée par une illégalité nommée par Martine Xiberras délinquance (1993, p. 121). La dérive à laquelle le personnage se laisse aller au début du roman est à vrai dire due à la violence dont lui-même se croit victime de la part des instances dirigeantes. Situation tendancieuse que celle-là : l'institué, rigide et dans une certaine mesure sclérosé, dresse une barrière destinée à retenir les élans d'éventuels instituants désireux d'en contester la mainmise. Moteur de tensions dans toutes les sociétés policées, ce dynamisme crée des oppositions génératrices d'agressivité, qui peuvent pousser l'État, maître des codes en vigueur, à intensifier les interdits restreignant la libération des pulsions individuelles et collectives. « La surrationalisation de cette structure sociale s'achève dans un irrationalisme complet et terrifiant renvoyant les individus à la plus " primitive" des angoisses », signale Michel Maffesoli (1984, p. 18). Résultat : une montée de violence insidieuse qui s'accentue à mesure que l'institution essaie de la maîtriser et dont les effets peuvent s'avérer funestes 
JEAN-PIERRE THOMAS, « Die With Your Boots On : agressivité et exclusion dans La rage, de Louis Hamelin »

pour le corps social. Le caractère fondateur d'une violence sainement évacuée se trouve dès lors nié.

Malarmé ne manque pas de considérer les institutions oppressives d'un mauvais œil. C'est d'ailleurs de regards dont il est question : « Le regard de la société, qui définit la catégorie de la déviance. Le regard des stigmatisés, qui intègre l'étiquette apposée par la société, mais qui développe néanmoins son propre point de vue. »(Xiberras, 1993, p. 96) Malarmé se définit comme un stigmatisé-transgresseur, mais sa voix résonne d'un faible écho contre le vacarme d'une machine étatique dont les symboles dans le roman sont les Jumbo Jets qui écrasent les habitants de la région « de leur rugissement et de leurs traînées laiteuses. » (17) J'ai mentionné l'importance accordée aux bruits dans La rage. Ceux-ci prennent souvent l'aspect du son tonitruant que font ces Boeing et l'un des remèdes contre ces bruits consiste à leur en opposer de meilleurs, sous forme de la musique qui jaillit des haut-parleurs du stéréo de Johnny. Le metal devient ainsi une arme instituant un nouveau système de valeurs fondé sur la nécessité d'opposer au code social en place un point de vue antagoniste afin d'orienter l'attention vers les inégalités prévalant. Ce système conquiert peu à peu Malarmé et permet à « la violence libérée, c'est-à-dire la révolte, [de] s'oppose[r] à la violence instituée, c'est-à-dire à la force légitimée. » (Balandier, 1979, p. 14) Pourtant, comme nous le verrons sous peu, aucune de ces deux formes de violence n'est fondatrice d'un nouvel ordre; leur visée commune est la destruction unilatérale à seule fin de persister.

Aux tentatives des agents fédéraux de déposséder les habitants de la région de leurs terres - l'expropriation a été relevée par plusieurs comme étant le thème principal du 
www.revue-analyses.org, vol. 14, n², automne-hiver 2019, p. 86-111.

roman $^{18}$-, le narrateur oppose une attitude déviante qui le conduit à recourir à la violence il prend le contrôle de la tour de contrôle de Mirabel. Coincé dans un univers d'antithèses qui briment sa liberté, il est de plus en plus poussé vers l'exclusion ${ }^{19}$, lui qui se définit par ses ennemis, reflet d'un système de pensée binaire (224). C'est ici que le message livré par les morceaux cités dans le roman prend son sens : l'appel à la liberté prôné par les groupes metal catalyse le désir de Malarmé d'être son propre maître. Il semble toutefois que le thème du chaos prime ici la dimension dionysiaque, ce qui limite le recours à la violence à la dimension destructrice de celle-ci. Comme le dit Burné, autre ami de Malarmé : «La seule façon de reprendre le contrôle de moi-même, c'est en me détruisant ». (170) Il ne paraît pas y avoir d'issue à l'atomisation forcée par l'institué réifiant, sinon dans l'appropriation du bien d'autrui, d'où la situation de squatter de Malarmé. La menace d'expropriation demeure toutefois présente, rappel de la toute-puissance de l'institué. Le monde est devenu pour l'individu un lieu lui étant étranger. S'il faut se frapper les balles sur le mur ou assumer une santé qui relève de la folie, c'est que l'univers aseptisé des dirigeants ne sied plus à une jeunesse qui rêve de vie au présent. Les représentants de l'institué orientent les énergies « en fonction d'un futur radieux, de lendemains qui chantent ou d'un paradis de transparence. Mais, face à cette propension institutionnelle, il y a les situations existentielles qui assument, consciemment ou pas, la circularité, le déjà-vu, le déjà-dit » (Maffesoli, 1984, p. 53), il y a une jeunesse qui juge utile de garder vivant le sociétal. La rébellion trouve sa raison d'être dans cet affrontement entre volonté et nécessité. Se considérant comme un parasite, Malarmé a «pris le parti de tout refuser»

\footnotetext{
${ }^{18}$ Julien Desrochers parle à ce titre d'une « double expropriation : celle de la jeunesse (la génération X) qui se voit dépossédée de ses ambitions et qui est aliénée par une société utilitariste, et celle des agriculteurs, expulsés de leurs terres par le gouvernement fédéral afin de permettre la construction de l'aéroport de Mirabel. » (2006, p. 70)

${ }^{19}$ David De La Roche voit en Malarmé un personnage qui « recherch[e] l'exclusion, pour ne pas être inclus dans un système normatif qu'il juge avilissant et aliénant. » (2006, p. 36)
} 
(366), conscient qu'à la volonté des tenants du système ne peut s'opposer que la nécessité de liberté. «Soudain j'ai été saisi d'une grande colère inutile, au hasard, poussant un cri étouffant au fond de ma gorge, $[\ldots]$ une colère qui ne demandait qu'une prise en charge à fond de train pour aboyer souverainement»(262), déclare le personnage. Inutile mais nécessaire, cette colère peut-elle résulter en une mobilisation d'énergies destructrices dont le but réel serait l'instauration d'un nouvel ordre?

Édouard Malarmé s'enfonce toujours davantage dans la désillusion et la réclusion. Ayant rompu avec les normes, il en vient à ne plus savoir qui il est.

Je veux être capitaliste, fasciste, socialiste, communiste, babouviste, stakhanoviste, libéral, conservateur [...], je suis déviant, radical, révisionniste, orthodoxe, intransigeant, hétérodoxe, éclectique et syncrétique, je suis tout en même temps, je me tape la surdose des doctrines. (195)

Malarmé ne peut que se trouver marginalisé puisque, embrassant tous les systèmes, il ne convient à aucun. Cette situation signale un «échec à la normalité [qui] semble constitutif des processus d'exclusion. » (Xiberras, 1993, p. 26) Mais comme les exclus « ressentent avec plus d'acuité l'essentielle désagrégation de la trame sociale » (Maffesoli, 1984, 72), Malarmé est perçu comme un danger pour les institutions, dans la mesure où il résiste à leur rigidité mortifère. Son discours qui prêche la rébellion représente une menace concrète aux yeux du groupe. Cette harangue transgressive relaie du reste les extraits de chansons heavy metal après les premiers chapitres - elle en est en fait le prolongement, reprenant à son compte le message transmis et l'amplifiant dans les plans délictueux du personnage. Le refus affiché a beau adopter dans un premier temps la simple forme d'un désir d'insoumission, il traduit rapidement l'ampleur de l'anomie qui frappera la société s'il est laissé libre cours à la violence. Le roman s'achève d'ailleurs dans la destruction. Porteur de différences par rapport à son groupe d'appartenance, Malarmé s'est graduellement 
www.revue-analyses.org, vol. 14, n², automne-hiver 2019, p. 86-111.

transformé en ce que les paroles des chansons qu'il écoutait au début du roman annonçaient. Si les extraits de pièces diminuent de façon notoire à mesure que l'intrigue se développe, c’est que le narrateur, par ses agissements et ses pensées, les remplace. Le roman se fait l'emblème de la lutte d'une jeune génération privée de sentiment d'appartenance à l'égard des institutions monolithiques qui la contrôlent.

\section{Heavy metal et littérature : de sains rapports de voisinage?}

Un débat de société intéressant s'est levé au cœur des années 1980 pour déterminer si la musique heavy metal incite ses adeptes à adopter des comportements violents. Ce débat fait toujours rage - quoique un peu plus en catimini aujourd'hui - et malgré qu'il ne soit pas question de prendre position ici, convenons que la perpétuation de cette controverse rend vivement compte de son acuité. Louis Hamelin semble avoir posé un constat équivalent vers la fin des années 1980. Il a toutefois pris parti : le visage qu'il présente du metal n'est pas des plus positifs. La violence banale, l'«atmosphère d'agressivité mesquine et quotidienne » (Xiberras, 1993, p. 127) qui marque le recours au metal dans le roman ne laisse que peu de chance à une violence fondatrice d'apparaître. Dans un ordre d'idées semblable, les noms de groupes inventés par l'auteur, Raging Dogs et Brains Blasters, connotent une animosité à l'état brut. Comment expliquer ces choix radicaux? S'agit-il bien au demeurant de choix? Hamelin, en 1989, fait partie d'une jeune génération d'auteurs qui semblent dire non au monde qui les entoure, mais qui n'ont pas de solution de rechange à proposer. La littérature leur sert d'exutoire pour gérer leur rage. Pourquoi avoir amalgamé ici la musique heavy metal à la littérature? D'aucuns n'hésiteraient pas à proclamer que le metal est un pur produit de la postmodernité. Éclaté, débridé, enragé mais à la fois promoteur de libertés inédites, il lance l'idée de possibilités audacieuses, dont l'auteur a en partie tiré profit. 
Sur le plan de la forme, l'emploi constant de passages écrits en lettres majuscules permet à Hamelin de hachurer son récit, ce qui le dote d'un rythme accidenté, brusque, voire violent. La lecture de l'œuvre se fait par à-coups, le lecteur butant sur des segments (onomatopées ou extraits de pièces musicales) qui représentent autant de barrières à franchir pour parvenir au dénouement de l'intrigue. L'entremêlement des actions et des paroles de chansons rend la lecture du texte périlleuse - ne comprend vraiment que celui qui décode la teneur des passages cités, et encore. En tant que renvoi porteur d'un sens précis, l'hypotexte musical interpelle le lecteur et en nourrit la perception, superposant les niveaux de lecture à même la phrase. Le fil de l'intrigue, discontinu, devient lui-même violence faite au lecteur. Le roman se terminera sur une onomatopée évoquant le langage codé et fragmenté des transmissions aéroportuaires, projetant la conclusion dans une sorte d'ailleurs langagier mystérieux - violence est ainsi faite au code langagier traditionnel. Le personnage de Malarmé ne peut apparaître dans ce contexte que morcelé, violenté luimême dans son être porteur de violence.

Les paroles de chansons interfèrent donc avec le récit au point de lui donner un tour insoupçonné. Les jeux de mots créés entraînent le lecteur sur des pistes de réflexion parfois complexes, qui l'invitent à établir des liens entre divers champs sémantiques. Dans «BACK BLACK BARDOT BIEN BLANCHE BIEN BAISANTE BIEN BANQUISE » (163), l'association de l'actrice française Brigitte Bardot au «Back in Black» d'AC/DC, antithèse frappante, donne un mélange explosif qui accentue la fragmentation du réel déjà évoquée au long de l'œuvre. La rage dont le roman raconte la montée est ressentie par le lecteur dans ces passages sur lesquels il bute sans cesse. Des formules comme «BACK BLACK BEAR OH SAY LIKE AN EAGLE AS HIGH AS THE BELLS BAT» ou 
www.revue-analyses.org, vol. 14, n², automne-hiver 2019, p. 86-111.

«BALLS TO THE BELLS MAN » (57) favorisent à la fois la déconstruction et la reconstruction du message des groupes metal afin d'en faire une litanie constamment renouvelée et, du coup, dotée d'une nouvelle efficacité. La lecture tangue de pair avec l'intrigue nourrie de rythmes trépidants.

Il semble, au vu du travail accompli par Louis Hamelin dans La rage, que la musique heavy metal sied à certains sujets. La tonalité violente associée à ce genre musical ainsi que les topoï qui l'accompagnent conviennent au récit. «[Heavy metal's] themes are replete with rebellion and general revulsion against the standards of polite society, and they emphasize the chaos which society seeks to keep at bay. $»^{20}$ (Weinstein, 2000, p. 249) La rage ressentie par Édouard Malarmé et la rage qui envahit simultanément son corps doivent-elles être vues comme le soubassement d'une catharsis saine? Ce ne semble pas être le cas dans La rage, où leur mise en relation avec le heavy metal confine au registre de l'exclusion. Hamelin s'est servi de thèmes précis (la révolte, la mort, la sexualité) au détriment de la posture idéologique intégrale adoptée par les adeptes de metal - « Parler de musique métal, c'est parler d'une musique offrant la dynamique de l'espoir dans une esthétique de refus », estime Martin Sasseville (2004, p. 308). Si le metal entend chanter la vie telle qu'elle est, dans son ambivalence foncière, sans en retrancher les aspects problématiques, conviant ainsi l'auditeur à en prendre en compte les diverses tonalités, Louis Hamelin a pour sa part choisi de mettre l'accent sur la dimension transgressive du genre, ce dont son roman profite certes.

\footnotetext{
${ }^{20}$ Je traduis : « Les thèmes privilégiés dans la musique heavy metal ont trait à la rébellion et au refus général de la société et ils soulignent le chaos dont celle-ci tente de se protéger. »
} 
JEAN-PIERRE THOMAS, « Die With Your Boots On : agressivité et exclusion dans La rage, de Louis Hamelin »

\section{Bibliographie}

ARNeTt, Jeffrey Jensen (1996), Metalheads. Heavy Metal Music and Adolescent Alienation, Boulder, Westview Press.

BALANDIER, Georges (1979), «Violence et anthropologie », Violence et transgression, Paris, Éditions Anthropos, p. 9-22.

Berger, Harris M (1999), Metal, Rock, and Jazz. Perception and the Phenomenology of Musical Experience, Hanover et Londres, Wesleyan University Press.

De LA Roche, David. 2006, Figures du marginal: Édouard Malarmé dans La rage de Louis Hamelin suivi de Couteau et autres incantations, Mémoire de maîtrise, Sherbrooke, Université de Sherbrooke.

DeSROChERS, Julien (2006), La rage de Louis Hamelin et le paradoxe sociocritique, Mémoire de maîtrise, Québec, Université Laval.

DURAND, Gilbert (1979), "Structure religieuse de la transgression», dans Michel Maffesoli et André Bruston (dir.), Violence et transgression, Paris, Éditions Anthropos, p. 23-33.

HAMElin, Louis (1989), La rage, Montréal, Éditions Québec / Amérique, Coll. « Littérature d'Amérique ».

HeIn, Fabien (2004), Hard Rock, Heavy Metal, Metal... Histoire, cultures et pratiquants, Nantes et Paris, Mélanie Séteun et Irma, Coll. « Musique et société ».

KAHN-HARRIS, Keith (2007), Extreme Metal. Music and Culture on the Edge, Oxford, Berg.

LAmONTAGNe, André (2004), Le roman québécois contemporain. Les voix sous les mots, [s.1.], Éditions Fides.

MAfFeSOl, Michel (1984), Essais sur la violence banale et fondatrice, Paris, Librairie des Méridiens.

MÉNARD, Valérie (2004), L'influence de Réjean Ducharme chez les écrivains de la génération X, Mémoire de maîtrise, Montréal, Université McGill.

Nareau, Michel et Jacques Pelletier (2015), «L'écriture comme appropriation de soi et du monde », Voix et images, vol. 41, n 1 (121), p. 7-11.

OuElLet, François et François PARÉ (2008), Louis Hamelin et ses doubles, [s.1.], Éditions Nota Bene. 
www.revue-analyses.org, vol. 14, n², automne-hiver 2019, p. 86-111.

SASSEVILLE, Martin (2004), «Pathologie d'une sous-culture urbaine en région. Comment le diable et sa musique officielle se débrouillent en région éloignée », dans Pierre-W. Boudreault et Michel Parazelli (dir.), L’imaginaire urbain et les jeunes. La ville comme espace d'expériences identitaires et créatrices, Sainte-Foy, Presses de l’Université du Québec, p. 307-320.

Stenning, Paul (2006), Iron Maiden. 30 Years of the Beast, New Malden, Chrome Dreams.

WALSER, Robert (1993), Running with the Devil. Power, Gender, and Madness in Heavy Metal Music, Middletown, Wesleyan University Press.

Weinstein, Deena (2000), Heavy Metal. The Music and its Culture, [Boston], Da Capo Press.

Xiberras, Martine (1993), Les théories de l'exclusion. Pour une construction de l’imaginaire de la déviance, Paris, Méridiens Klincksieck.

\section{Notice biobibliographique}

Jean-Pierre Thomas est professeur agrégé au campus Glendon de l'Université York, à Toronto, où il enseigne la littérature québécoise et la langue française depuis 2005. Il est spécialisé dans la recherche et l'étude de traces de mythes au sein des œuvres de la littérature québécoise des XIXe et XXe siècles et a publié des articles sur le sujet dans des revues nord-américaines et européennes. 